\title{
How to Prepare a Patient for Transarterial Radioembolization? A Practical Guide
}

\author{
Alban Denys • Marc Pracht • Rafael Duran • \\ Boris Guiu - Salim Adib · Ariane Boubaker • \\ Pierre Bize
}

Received: 18 November 2014/Accepted: 2 February 2015/Published online: 1 April 2015

(C) Springer Science+Business Media New York and the Cardiovascular and Interventional Radiological Society of Europe (CIRSE) 2015

\begin{abstract}
Transarterial radioembolization consist in delivering small particles loaded with Yttrium90, a pure beta emitter, in order to treat primary and secondary liver tumors. This treatment needs precaution since inadequate delivery of these beads can lead to severe complications. Moreover, a precise radiation dosing is crucial to achieve a good clinical success and to avoid complications such as radioembolization-induced liver disease. This review describes all the precautions and highlights clinical and technical considerations that need to be taken to avoid complications.
\end{abstract}

Keywords Liver tumor - Embolization .

Radioembolization · Y-90

A. Denys $(\bowtie) \cdot$ B. Guiu · P. Bize

Department of Radiology and Interventional Radiology, Centre Hospitalier Universitaire Vaudois and University of Lausanne, Rue du Bugnon 46, 1011 Lausanne, Switzerland e-mail: Alban.Denys@chuv.ch

\section{Pracht}

Department of Oncology, Centre Hospitalier Universitaire

Vaudois and University of Lausanne, 1011 Lausanne,

Switzerland

R. Duran

Department of Interventional Radiology, John Hopkins

University, Baltimore, MD, USA

S. Adib - A. Boubaker

Department of Nuclear Medicine, Centre Hospitalier

Universitaire Vaudois and University of Lausanne,

1011 Lausanne, Switzerland

\section{Introduction}

During the last decades, several transarterial locoregional therapies have been developed to treat liver cancer. Among them, transarterial radioembolization (TARE) has grown into a recognized palliative treatment option for patients with primary or secondary liver cancers. TARE has been initially developed in 1967 for the treatment of lung metastases [1] and came for liver clinical application in the 90 ' with the first human phase 1 escalation dose and phase 2 studies [2-4]. The concept is rather simple. The locoregional delivery through the hepatic artery of small particles of 50 microns loaded with Yttrium $90\left({ }^{90} \mathrm{Y}\right)$, a pure beta emitter, relies on the predominant arterial supply to the liver tumors in comparison to the adjacent normal liver who receives mainly blood flow from the portal vein [5]. Thus, high radiation dose can be delivered to the tumor and a limited dose to the normal parenchyma. Recent studies performed on hepatic specimens analyzed after radioembolization [6] showed that most of the changes in the tumor tissue were consistent with a direct radiation effect with a limited immune response. Thus, in order to ensure proper treatment delivery and prevent potential complications related to nontargeted administration, a careful pre-treatment preparation is crucial. Dose calculation and patients' follow-up need close collaboration between oncologists, nuclear medicine specialists, and interventional radiologists. The aim of this article is to give an overview of the practical issues raised by this technique and how to solve them.

\section{Transarterial Radioembolization Overview}

TARE is a two-step procedure: the preparation/simulation phase and the treatment phase. In the former, the patient 
comes as an outpatient or is admitted overnight depending on the centers' preferences and resources. A simulation angiogram is performed for two reasons. The first reason is to identify the arterial anatomy of the liver, potential arterial variants, tumor feeders, and extrahepatic branches coming off the hepatic arteries which might require proximal occlusion in order to avoid inadvertent microspheres delivery in these territories. The second reason is to predict the ultimate ${ }^{90} \mathrm{Y}$ deposition. Indeed in the same angiographic session, the position of the catheter that will be used for the treatment phase is decided (selective to tumor vasculature, segmental, lobar, or hepatic). Once the catheter in good position, a standard dose (50-180 MBq) of technetium-99 m-labeled macroaggregated albumin (Tc99-MAA) is slowly injected in close collaboration with colleagues from nuclear medicine in order to predict the TARE setting [7]. Planar and single-photon emission computed tomography are then obtained to measure the hepatopulmonary shunting, identify other potential extrahepatic deposits of Tc99-MAA, and to determine the captation ratio of the tumor relative to adjacent liver parenchyma. The dose of ${ }^{90} \mathrm{Y}$ to be delivered is then calculated using a specific formula (see below chapter 6).

Once the preparation/simulation phase is completed, the patient is rescheduled for the treatment phase (on average 1-2 weeks later) as an outpatient or for an overnight admission depending on the local expertise and regulatory issues. During a second hepatic angiography, the catheter is positioned in the exact position established during the simulation phase and the liver vasculature is again verified. Complementary embolization may be performed if needed. Glass (TheraSphere; Nordion, Ottawa, Ontario, Canada) or resin (SIR-Spheres; SIRTeX Medical, Lane Cove, NSW, Australia) microspheres are then slowly injected. After the procedure, a positron emission tomography (PET) or bremsstrahlung SPECT is performed the same day to verify ${ }^{90} \mathrm{Y}$ microsphere distribution in the liver [8].

\section{The Choice of the Material}

As previously mentioned, precise delineation of the arterial anatomy and evaluation of tumor vascular supply are critical to prevent potential severe complications, particularly in regards to the gastric, duodenum, or abdominal walls. To achieve this goal, the catheter's choice matters. Ideally the catheter should be as atraumatic as possible while allowing high-flow injection when needed to identify the vascular anatomy and its potential variants. A special attention should be directed toward arterial spasm which may modify hemodynamics downstream and consequently makes dose calculation impossible or false when using partition model (Fig. 1).
A combination of 4 or $5 \mathrm{~F}$ guiding catheter (cobra 2 or simmons 12 shape) and $2.8 \mathrm{~F}$ micro-catheter is recommended. This large lumen micro-catheter allows high-flow injection at 3 or even $3.5 \mathrm{cc} / \mathrm{s}$ that cannot be achieved with smaller lumen micro-catheters (even 2.7Fr does not allow more than $2 \mathrm{cc} / \mathrm{s}$ injection rate even at very high PSI injection pressure). For complex, distal embolization of small branches, a smaller angulated 2.0F micro-catheter may be used instead, if needed. Some authors recommend the use of a specially designed catheter: the surefire catheter (surefire medical, Westminster, CO, USA) which is a combination of a micro-catheter and expendable stent that is designed to prevent reflux of beads. This catheter has the possibility to reduce the procedure time by alleviating the need for collateral branches embolization, [9] (Fig. 2). It does, however, induce a significant blood pressure reduction downstream [10]. The consequence of this pressure change on the respective perfusion of the tumor and liver parenchyma has not been explored to our knowledge. A special attention to spasm and vascular damage risk along the Surefire catheter is also crucial.

\section{How to Solve Anatomical Variants of the Hepatic Arteries?}

Conventional anatomy is only encountered in $60 \%$ of patients with a single hepatic artery vascularizing the whole liver originating from the celiac trunk. This means that in $40 \%$ at least two different arteries vascularize the liver [11]. The most common variants are the so-called replaced right hepatic artery coming off the superior mesenteric artery (SMA) and the replaced left hepatic artery coming from the left gastric artery. If a patient has bilobar tumors and multiple hepatic arteries, there are two options. The first option is to redistribute the blood flow in order to have the whole liver vascularized by only one artery like for intra-arterial chemotherapy $[12,13]$. In a previous work published by Laenstein et al. flow redistribution allowed for the administration of particles in both lobes in a similar way in 24/27 patients [12]. Bilbao et al. looked at the same criteria in a similar group of 24 patients [13]. In their study, the distribution of Tc99-MAA was identical in both parts of the liver in only $66.6 \%$ of the patients. This means that a reduced dose is probably delivered in the redistributed part of the liver, but this did not translate in a reduced response rate to treatment in most cases (23/24 patients). One must also be aware that this technique of redistribution must be carefully done. Obstruction of the redistributed branch must be proximal to allow for the development of collateral branches, but any extrahepatic branch left patent distally to this occlusion will be hardly accessible in the future. This may not only preclude TARE in this territory but in the whole liver from the patent arteries (Fig. 3). 
A

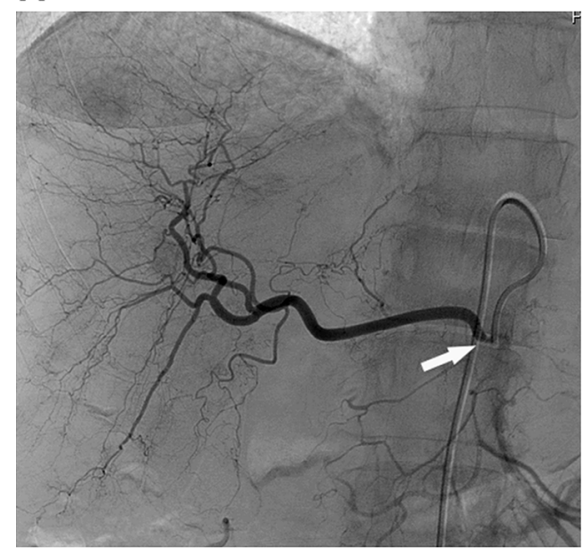

C

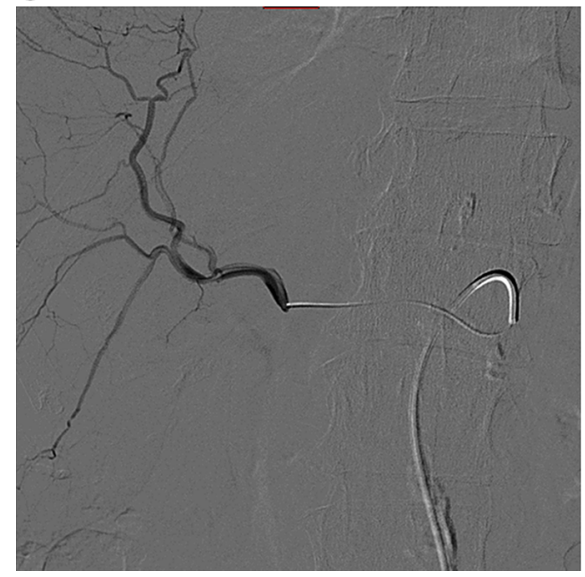

B

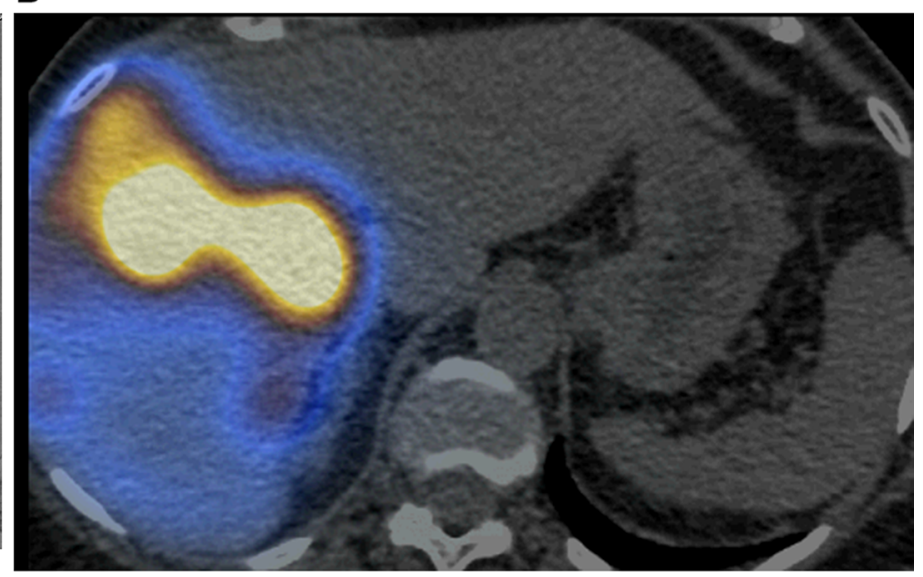

D

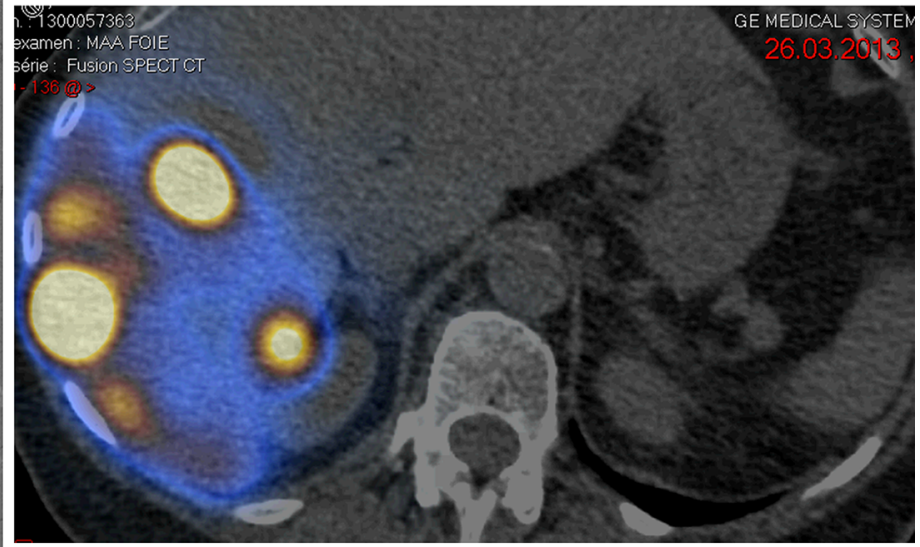

Fig. 1 72-year-old man with known uveal melanoma metastatic to the liver. A Initially, a replaced right hepatic artery was selected using a 5F Simmons 2 catheter followed by an angiography. Spasm at the origin of the artery is identified (white arrow). B Macroaggregated albumin (MAA) SPECT/CT showed that MAA particles were mainly delivered to the anterior sector of the right liver. C Repeated

In our practice, if the patient has 2 or 3 hepatic arteries, we prefer to perform TARE through each single one of them in order to keep all future options opened rather than redistributing blood flow. This makes the procedure more complex notably since radiation dose has to be calculated for each artery.

\section{How to Solve Tumor Feeding from Parasiting Arteries?}

Parasitized extrahepatic artery is defined by a flow to a hepatic tumor coming from an extrahepatic artery usually feeding adjacent structures. This is a frequent feature reported in up to $37 \%$ of cases when carefully searched by physicians. The most frequent one is the inferior phrenic artery [14] taking part of the vascularization of right-sided liver tumors. Other branches can also participate such as angiography was performed with the use of a $5 \mathrm{~F}$ Cobra as a guiding catheter in combination with a flexible $2.4 \mathrm{~F}$ micro-catheter with subsequent injection of Tc99-MAA. D The new MAA SPECT/CT showed an excellent tumor to liver ratio both in the anterior and posterior right liver sectors

intercostal, omental, and internal mammary arteries. These branches must be recognized before treatment. One way to identify a parasiting artery is to perform an arterial CT or a cone-beam CT with direct injection of contrast medium into the hepatic artery at the beginning of the procedure. Such techniques are useful in tumors located close to the hepatic dome or to the falciform ligament. By doing so it may be identified that part or most of the tumor vascular supply does actually not come from the hepatic artery itself. Two different solutions have been proposed by some authors. One is to embolize those parasiting arteries before TARE using particles and coils as proposed by Abdelmaksoud et al. [15]. This group demonstrated that the territory previously fed by the parasiting artery was re-perfused after TARE through intrahepatic or intratumoral collaterals by the hepatic artery in $96 \%$ of territories demonstrated on MAA scintigraphy [15]. Although we may assume that this redistribution will 
induce a homogenous distribution of microspheres in the tumor, this has never been demonstrated. The second solution is to perform TARE from these arteries when possible. Burgmans et al. recently demonstrated that TARE from the right inferior phrenic artery was feasible without complications or nontarget embolization [14]. In our practice, we have adopted the technique reported by Abdelmaksoud et al. [15] because of its simplicity. However, when facing complex situations, this technique might not be suitable. In postoperative patients, multiple adhesions between the liver and adjacent organs can occur. Parasiting arteries coming from the intestines (e.g., ileal or colic branches) may be tricky to deal with, particularly when multiple small branches vascularize the liver surface.

\section{How to Embolize Extrahepatic Branches of the Hepatic Artery?}

The embolization of extrahepatic branches remains a debated subject that is clearly related to the risk of delivering radioactive beads in digestive or pancreatic branches. This question posed for every single patient cannot be answered easily. The basics are that if there is a branch feeding an extrahepatic territory downstream of the final catheter position for beads injection, this branch must be embolized. This is particularly true for the right gastric artery which origin is usually located distally to the gastro-duodenal artery (GDA) [16]. The right gastric artery can be accessed both from the hepatic branches with a direct access along the arterial flow or in retrograde approach from the left gastric artery through the small curvature arcade of the stomach [17]. In our experience, although technically challenging, this latter approach is much less risky avoiding potential hepatic artery dissection during manipulation. The final position of the micro-catheter must be as close as possible to the hepatic artery in order to place the coil exactly at origin of the right gastric artery. Due to the small diameter of this small curvature arterial arcade, a 2.0F micro-catheter might be necessary to reach the hepatic side of the arcade from a left gastric artery approach. We also inject vasodilatator in the left gastric artery when starting the catheterization in order to avoid spasm in the small curvature arcade which may already have per se a very small diameter. This branch must be recognized in every case, otherwise extensive necrosis of the gastric wall may occur [18] (Fig. 4). Prophylactic embolization of the GDA is much more debated. At the beginning of TARE experience, this artery was embolized in every single case. The problem that appeared is that small collaterals from the hepatic artery usually in the liver hilum feeding the pancreatico-duodenal artery territory may increase in size between the
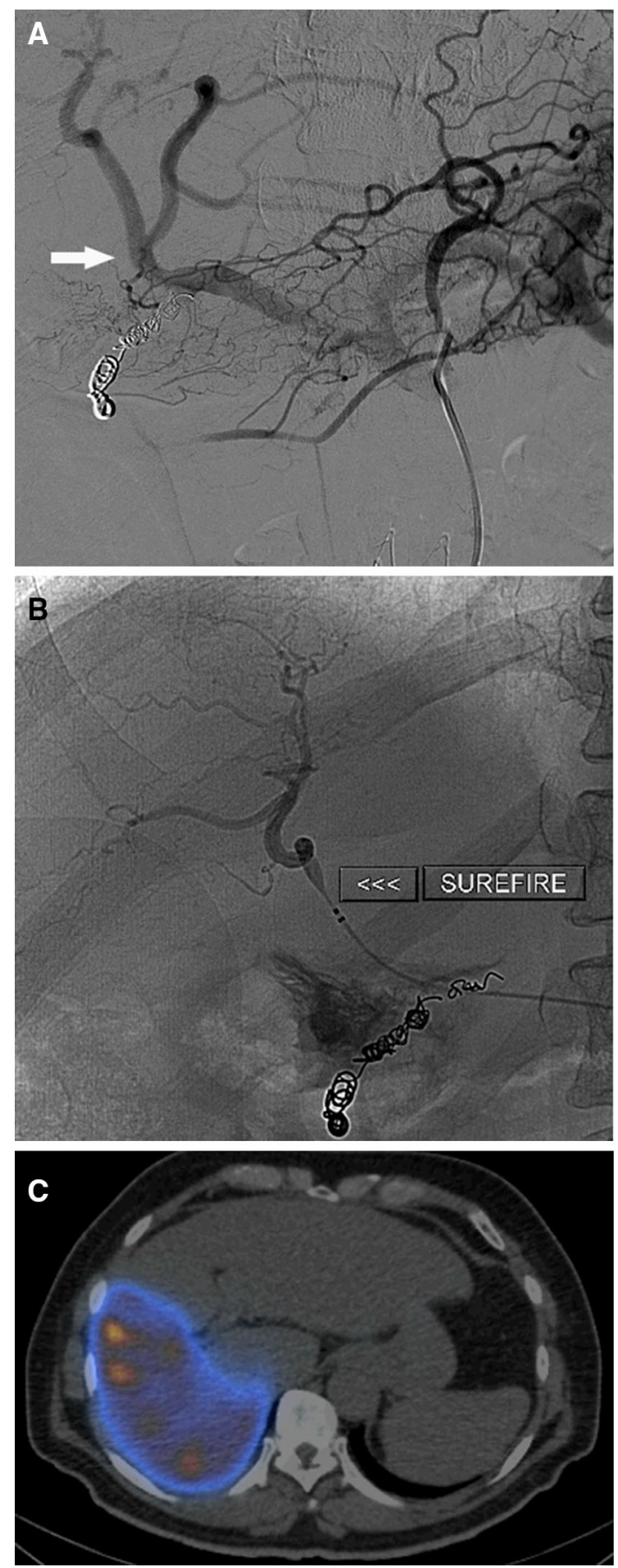

Fig. 2 57-year-old man with known uveal melanoma metastatic to the liver undergoing his first session of radioembolization to the right lobe. A Selective angiogram performed through the left gastric artery showed a tiny network of collaterals connecting the left gastric artery to small supraduodenal arteries (white arrow) originating at the hepatic artery bifurcation that developed after embolization of the GDA and right gastric artery in the preparation phase. These arteries could not be catheterized selectively from the left gastric artery nor the hepatic bifurcation. B A surefire catheter is deployed downstream of these branches to safely deliver the MAA particles. C SPECT/CT showed a distribution of the MAA in the right lobe without any captation of the gastric or duodenal wall 
A

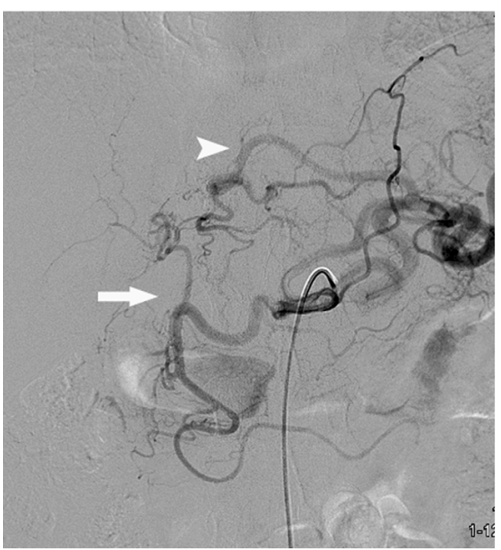

D

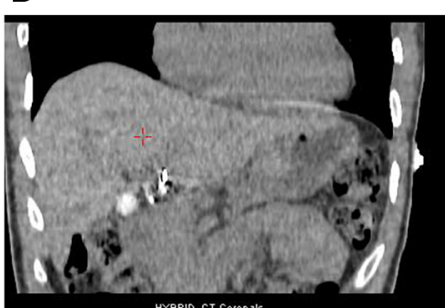

HYERIO_CT Coronals
B

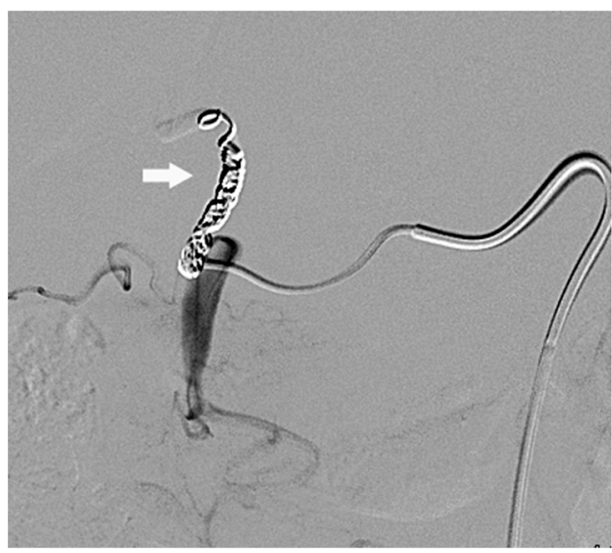

C

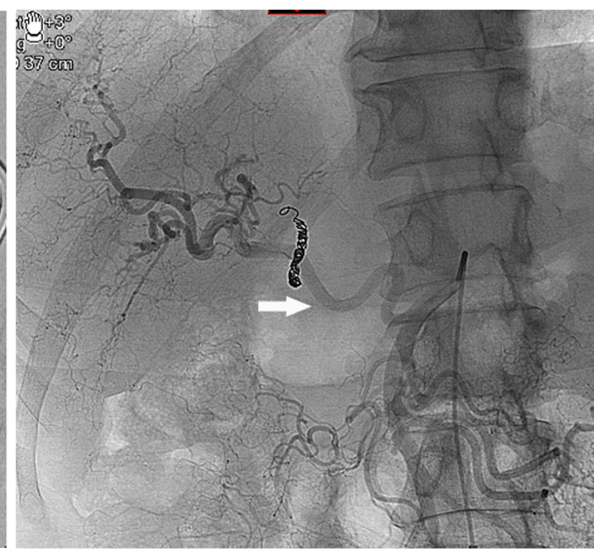

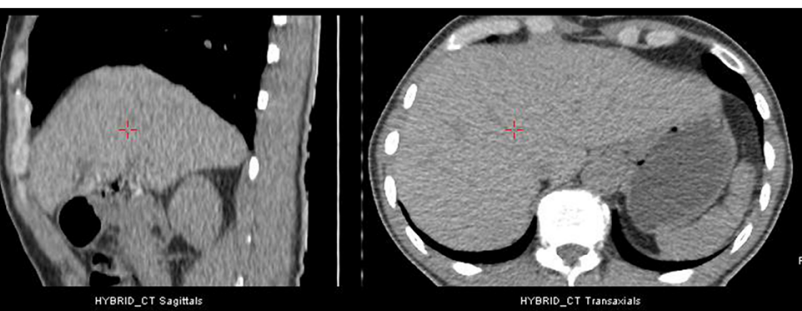
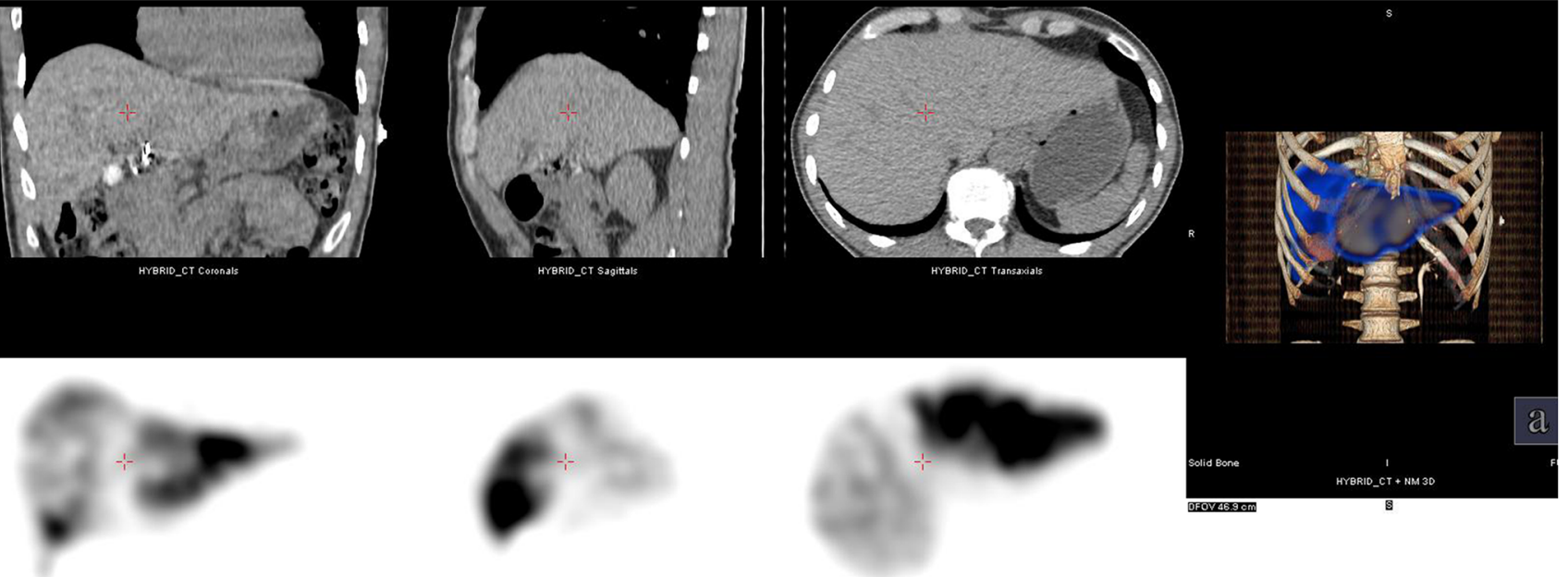

TFOVA890m

HYQRID_CT + NM 30

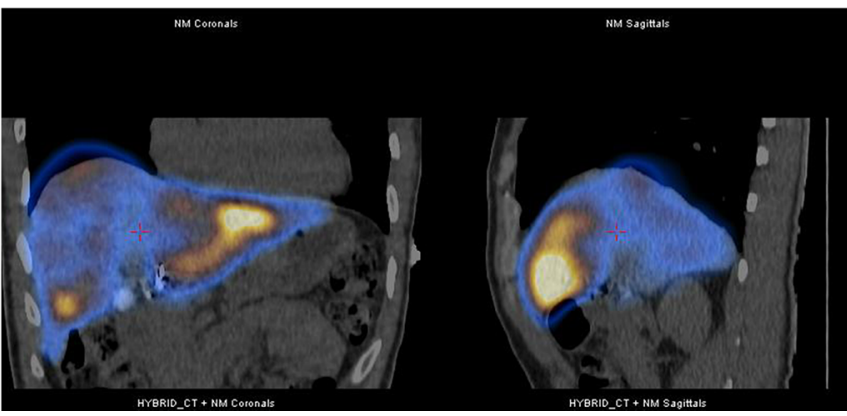

Fig. 3 66-year-old man with colon cancer and bilobar liver metastases selected for radioembolization. A Celiac angiogram shows a replaced left hepatic artery coming off the left gastric artery (white arrowhead) and a common hepatic artery (white arrow) of small size feeding segment 4. B Because of the small size of this common hepatic artery, it was embolized proximally using coils (white arrow). C Superior mesenteric angiography showed a large replaced right

preparation/simulation phase and the treatment session. These tiny branches are hardly embolized without risk and prolong the procedure time [19]. Also, these branches may be so small that precise coil embolization is impossible and other embolic agent, such as glue, must be used [20]. hepatic artery feeding the segments 5-8 of the liver. D After injection of Tc99-MAA alternatively in the replaced right and left hepatic arteries $2 \mathrm{~h}$ later, multiplanar reconstructions of the liver showed excellent distribution of MAA in the right segments (5-8) and in the left lobe (2-3) without Tc99-MAA activity in segment 4 due to previous coil embolization of the common hepatic artery and poor collateralization from either the right or left lobe arteries

Although this phenomenon is rarely observed (less than 10 cases out of 543 sessions in the Samuelson series), when present makes the TARE procedure much more complex [20]. More recently, Hamoui et al. demonstrated that embolization of the GDA is not mandatory as long as a 

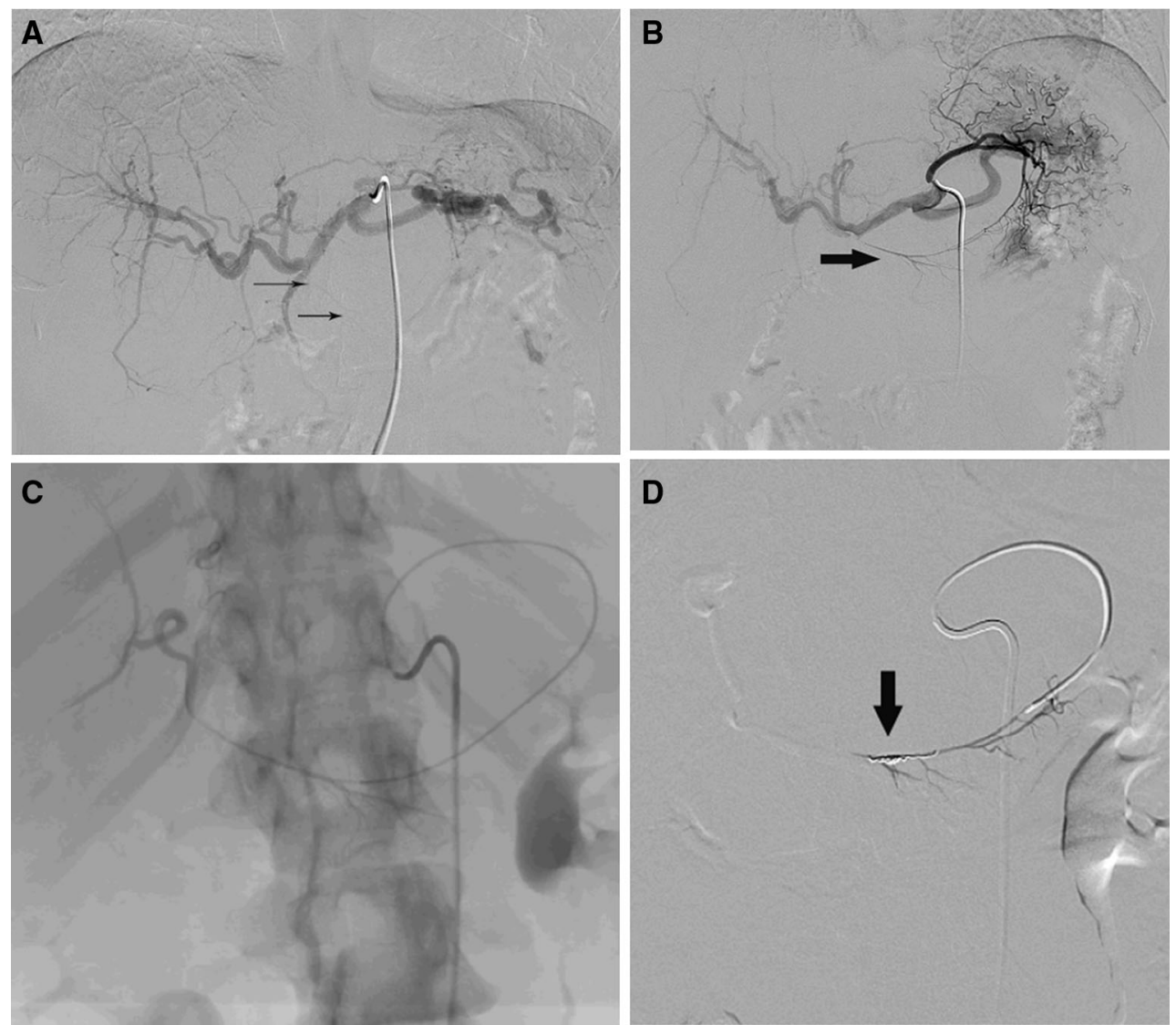

Fig. 4 45-year-old man with rectal cancer and bilobar liver metastases selected for radioembolization as a salvage therapy. A Celiac angiogram identified a very small right gastric artery coming off the arterial hepatic bifurcation (small arrows). B Selective left gastric angiogram showed the small curvature arcade connecting with the

distance of $2 \mathrm{~cm}$ is observed between this artery and the catheter in his final position for treatment delivery [16]. This is particularly true when using Therasphere particles which are less numerous and therefore less embolic minimizing the risk of reflux. In a recently published work, Lam et al. reviewed the causes of gastro-duodenal ulceration in a 247 cases series and found that stasis observed during TARE was the most significant risk factor [21].

A special attention must be paid to a rare anatomic variation of the gastro-hepatic arterial arcade: this variant consists in the vascularization of part of the stomach by the left hepatic artery through small branches passing through the ligamentum venosum. These branches are recognized by the fact that they do not give side branches until reaching the stomach unlike hepatic branches rapidly dividing in parenchymatous side branches (Fig. 5). A conebeam $\mathrm{CT}$ injection may also be helpful to demonstrate the anatomy.

Embolization of the cystic artery remains controversial. In order to prevent radiation cholecystitis, some authors right gastric artery (bold arrow). C Selective angiogram using a 2.0F micro-catheter showed filling of the hepatic artery. D Selective angiogram confirmed the occlusion of origin of the right gastric artery after embolization with 0.018 in microcoils using a retrograde approach

recommend the use of proximal cystic artery embolization either using coils or large pledgets of gelfoam. Interestingly, in this study, nearly $50 \%$ of their 46 patients had either recanalization or collateralization of the cystic artery at 1 month [22] (Fig. 6). This clearly raises doubts on the benefit of such procedure for a complication (i.e., radiation cholecystitis) reported in less than $1 \%$ of patients [23]. In our center, attention is paid not to place the catheter in front of the origin of the cystic artery in order to minimize the risk of combined ischemic and radiation-induced injury. And in all other cases, the cystic artery is left patent.

Falciform artery embolization is also a debated issue. This small artery feeding the faciform and round ligament up the anterior abdominal wall is sometimes recognized on angiogram with its characteristic course in the direction of the umbilicus and on Tc99-MAA SPECT/CT. The presence of this artery was recognized in a large series (224 procedures) in $9.3 \%$ of cases [24]. The same group did not take any prophylactic measure to prevent skin complications and actually did not observe any. Although rarely 

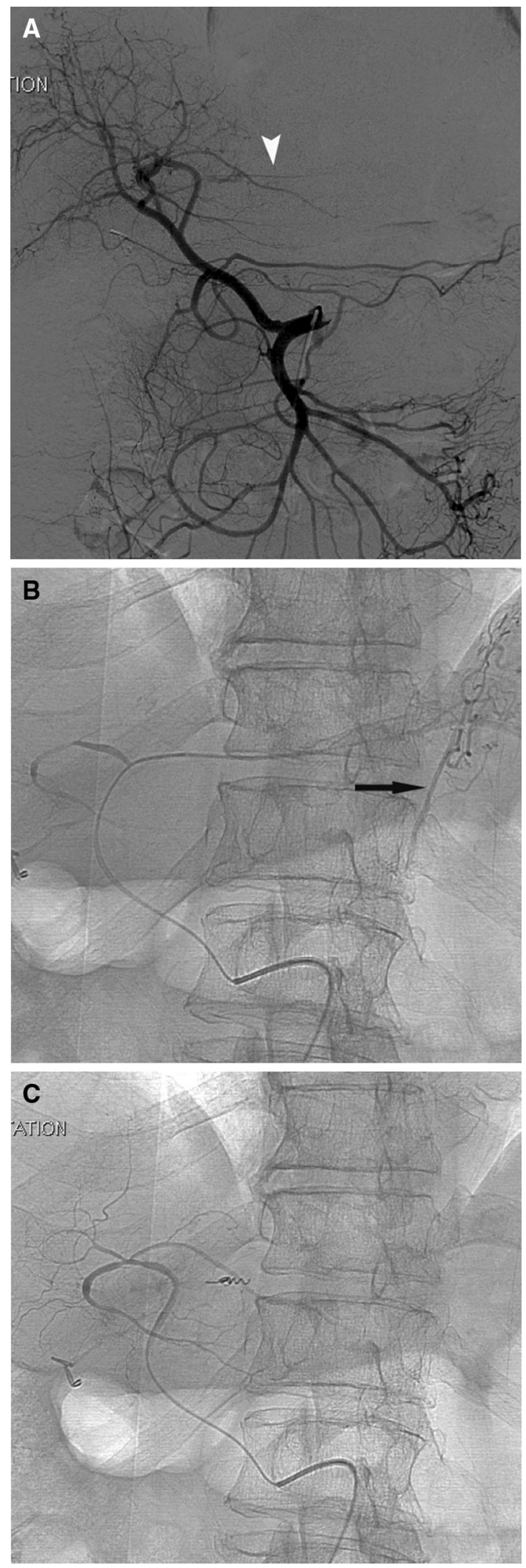

४Fig. 5 53-year-old man with neuroendocrine tumor and liver metastases selected for radioembolization as a salvage therapy. A Celiac angiogram showed a tiny branch coming off the left hepatic artery with a horizontal course (small white arrowhead). B Selective catheterization of this artery followed by angiography showed that was supplying the small curvature arcade giving multiple gastric branches (black arrow). Note that side branches were only visible coming off this small artery when leaving the ligamentum venosum. C After coil embolization, no gastric branches are visible during left hepatic arteriogram
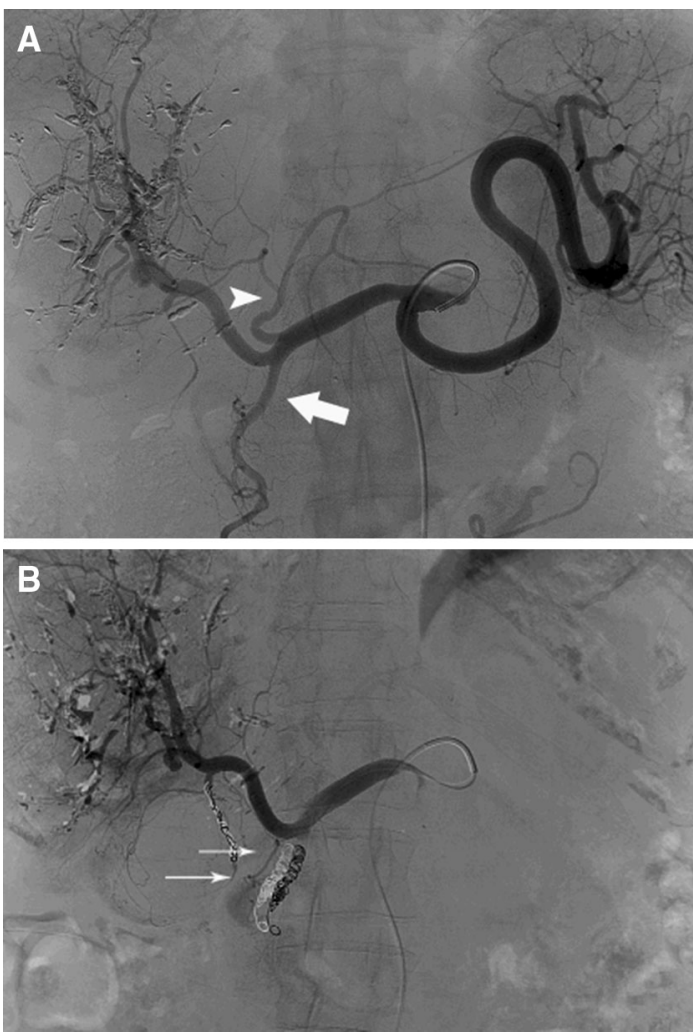

Fig. 6 A 57-year-old patient has multiple liver metastases from colorectal cancer in the right lobe of the liver. Due to insufficient growth of the left hepatic lobe after right portal embolization, a right hemihepatectomy was cancelled and TARE procedure of the right lobe decided. A Note that the left hepatic branch (arrow head) origin is upstream of the gastroduodenal artery (bold arrow). B After proximal embolization of the cystic and of the gastroduodenal artery, we observe an enlarged arterial network of very small arteries feeding the cystic wall and probably the choledochus

Table 1 Reduction in the prescribed activity of ${ }^{90} \mathrm{Y}$ resin microspheres to ensure safety of the lungs

\begin{tabular}{ll}
\hline Percent lung shunting & Activity of SIR-spheres microspheres \\
\hline$<10 \%$ & Deliver full amount of SIR-spheres \\
$10-15 \%$ & Reduce amount of SIR-spheres by $20 \%$ \\
$15-20 \%$ & Reduce amount of SIR-spheres by $40 \%$ \\
$>20 \%$ & Do not give SIR-spheres microspheres
\end{tabular}

According to the manufacturer instructions [41] 
Table 2 Properties of commercially available ${ }^{90} \mathrm{Y}$ microspheres medical devices

\begin{tabular}{lll}
\hline Description item & SIR-spheres ${ }^{\text {vr }}$ & TheraSphere $^{\text {VR }}$ \\
\hline Sphere material & Resin with bound yttrium & Glass with yttrium in matrix \\
Sphere diameter $(\mu \mathrm{m})$ & $20-60$ & $20-30$ \\
Activity in single vial $(\mathrm{GBq})$ & 3 & $3,5,7,10,15,20$ \\
Number of spheres per vial & $40-80 \times 10^{6}$ & $1.2-8 \times 10^{6}$ \\
Density $(\mathrm{g} / \mathrm{cm} 3)$ & 1.6 & 3.29 \\
Assumed activity per sphere $(\mathrm{Bq})$ & 50 & 2500 \\
Shelf life & 24 h after calibration & 12 days after calibration \\
${ }^{90}$ Y half-life & 2.67 days & 2.67 days \\
\hline
\end{tabular}

Modified from the recommendations of the American Association of Physicists in Medicine [42]

observed, severe cases of skin necrosis have been reported when no preventive measures were taken $[25,26]$. This artery can be embolized but its catheterization is not always easy and carries the risk of arterial spasm or dissection. A straight forward solution is to place topical ice around the umbilicus during treatment in order to induce locoregional vasoconstriction of the skin arteries. This simple method avoids complex catheterization and seems efficient in a limited series of 14 patients recently described by Wang et al. [27]. We have endorsed this technique, but we have to emphasize that the skin territory fed by this artery is variable in size and shape around the umbilicus. Topical ice placement should cover precisely those territories.

\section{How to Optimize Dose Calculation?}

Simulation study with Tc99-MAA injection is used to calculate the delivered dose [28]. Anatomic distribution of tumor as well as the functional capacity (limited hepatic reserve due to cirrhosis, previous chemoembolization, exposure to systemic chemotherapy or biologic agents, etc.), whole liver, unilobar, or segmental approaches must be taken into consideration.

Irrespective of the location of Tc99-MAA injection (between 50 and $180 \mathrm{MBq}$ depending on the injected liver volume), it is imperative that the MAA being delivered with flow rates and catheter position that mimic the anticipated ${ }^{90} \mathrm{Y}$ infusion rate.

Scintigraphy should be performed within $1 \mathrm{~h}$ of injection of Tc99-MAA to prevent false-positive extrahepatic activity due to free technetium (administration of thyroid blocking agent such as $400-600 \mathrm{mg}$ perchlorate is recommended). Whole-body planar scintigraphy and hepatic SPECT/CT imaging are performed to calculate the lung shunt fraction (i.e., percentage of MAA going to the lung), to exclude any extrahepatic deposition in the duodenum or stomach, and to depict intrahepatic radiopharmaceutical distribution (ratio of MAA going to the tumor vs normal liver). At low radiation dose levels, the amount of radiation shunted to the lung is acceptable: less than $20 \%$ of lung shunting for SIR-Spheres (the amount of ${ }^{90} \mathrm{Y}$ should be reduced if the percentage of lung shunting is greater than $10 \%$, see Table 1), and less than 30 Gy for TheraSphere. Radiation damage to the lungs is cumulative, so repeated use of microspheres may lead to radiation pneumonitis (maximal cumulative dose to lungs: $50 \mathrm{~Gy}$ ).

SPECT/CT images are analyzed to verify the correspondence between the lesions documented by CT, MRI or $\mathrm{PET} / \mathrm{CT}$, and regions with high Tc99-MAA uptake, to evaluate the tumor to non-tumor ratio $(\mathrm{T} / \mathrm{N})$ for the most representative lesions and for volumetric purposes (tumor and nontumoral volumes). In case of poor correlation with lesions due to technical problems, such as, for example, a preferential blood flow due to the catheter position in the artery, the simulation study may be repeated.

The dosimetry calculation depends on the beads to be injected. The microspheres are loaded with the same highenergy $\beta$-emitter radioisotope ${ }^{90} \mathrm{Y}$, having optimal characteristics for therapy (half-life: $64.2 \mathrm{~h}$; average energy: $0.93 \mathrm{MeV}$; maximum and mean penetration ranges in soft tissue: 11 and $4 \mathrm{~mm}$, respectively) [29]. Currently, two ${ }^{90} \mathrm{Y}$-microsphere products are available commercially worldwide: one is composed of ${ }^{90} \mathrm{Y}$-doped resin (SIRSpheres ${ }^{\text {VR }}$; Sirtex Medical Limited, North Sydney, Australia) and the other one incorporates ${ }^{90} \mathrm{Y}$ in a glass matrix (TheraSphere ${ }^{\mathrm{VR}}$; Nordion Inc., Ottawa, Canada) (Table 2).

The activity planning methods and dose toxicity for TARE differ fundamentally from those for external beam radiotherapy, or point source brachytherapy. Although one activity calculation method has been proposed for ${ }^{90} \mathrm{Y}$ glass microspheres, three alternatives exist for resin microspheres. Recommendations in liver lobar TARE are different in terms of maximum tolerable liver absorbed dose according to the medical device: less than $70 \mathrm{~Gy}$ when using resin microspheres [28] and less than 120 Gy with glass microspheres [30]. 
Table 3 Risk factors for REILD

Total bilirubin level $>1.2 \mathrm{mg} / \mathrm{dL}$ or $>20 \mu \mathrm{mol} / \mathrm{L}$, especially if cirrhosis

Whole-liver treatment

Activity higher than $0.8 \mathrm{GBq} / \mathrm{L}$ of targeted liver

Previous and/or subsequent ( $<2$ months) intravenous or intraarterial chemotherapy, especially if no cirrhosis

Small liver volume $(<1.5 \mathrm{~L})$ or huge tumor involvement $(<70 \%$ of the liver)

High dose delivered to the targeted liver ( $\geq 150 \mathrm{~Gy}$ ) or to the nontumoral liver ( $>52$ Gy? $>37$ Gy?)

Young people ( $<45$ year)

Sequential lobar treatment if repeated with an interval of less than 6 weeks

\section{${ }^{90} \mathrm{Y}$ Resin Microsphere SIR-Spheres}

The so-called "empiric method" recommends a standard amount of activity, which is varied only according to the size of the tumor within the liver. It requires CT scan or MRI to estimate the liver and tumor sizes. This model has suggested administration of $2.0 \mathrm{GBq}$ for $<25 \%$ of tumor involvement of the liver, $2.5 \mathrm{GBq}$ for $25-50 \%$ of tumor involvement, and 3.0 GBq for $>50 \%$ of tumor involvement. This method is actually not recommended due to a high rate of toxicity [28]. Kennedy et al. [31] reported $4 \%$ of radiation-induced liver disease (RILD) after TARE, $75 \%$ of them from one center, which used the empiric method.

The body surface area (BSA) is the most widely used method. It is a variant of the empiric model where implanted activity is adjusted according to the size of the tumor within the liver and the size of the patient. This model assumes that the size of each patient's liver correlates with the BSA. This calculation requires the liver and tumor volume from the CT scan or MRI.

Activity $(\mathrm{GBq})$ for a whole-liver treatment

$=(\mathrm{BSA}-0.2)+[$ volume of tumor/(volume of tumor + volume of nontumoral liver)].

The BSA method determines the maximum activity that can safely be implanted subject to the limitations imposed by lung shunting and maximum tolerable dose to the normal liver. It is simple and user friendly, and recommended for use in sub-centimeter tumors or tumors with ill-defined margins, by visual estimation of the overall tumor burden. However, it provides no information about actual dosimetry to tumor and normal or nontumoral liver, artificially limits the injected ${ }^{90} \mathrm{Y}$ activity between 1.0 and 3.0 GBq and may cause over- or under-treatment in selected patients [32].

The Partition Model [32] can only be used where the tumor mass is localized in a discrete area within the liver and the tumor can be drawn as an "area-of-interest" on a SPECT camera image.

$$
\begin{aligned}
& \text { Activity }(\mathrm{GBq})=\left[D_{\text {Liver }}(\mathrm{Gy})\right. \\
& \left.\quad \times\left(\left(T / N M_{\text {Tumor }}\right)+M_{\text {Liver }}\right)\right] /[49670(1-L / 100)]
\end{aligned}
$$

with $L=$ the percentage lung shunting, $T=$ tumor, $N=$ normal liver, $M=$ mass.

This model is more complex with less historical clinical data as compared to the BSA method. However, it allows personalized dosimetry with a greater physician control over radiation doses (Gy) to lung, liver and tumor compartments, and no theoretical limit to the ${ }^{90} \mathrm{Y}$ - activity to be injected. The desired ${ }^{90} \mathrm{Y}$ activity in cases of high hepatopulmonary shunting is adjusted on the basis of predicted lung radiation dose (Gy) [32].

\section{${ }^{90} \mathrm{Y}$ Glass Microspheres TheraSphere}

The dosimetry recommended by the manufacturer is based on the MIRD (Medical Internal Radiation Dosimetry) model, assuming homogeneous activity distribution in the liver, tumor, and lung (if any shunting occurs). Activity calculation requires the patient's perfused liver mass (lobar or segmental) and the nominal target dose.

$$
\begin{aligned}
\operatorname{Activity}(\mathrm{GBq})= & \operatorname{Dose}(\mathrm{Gy}) \\
& \times \text { Perfused liver mass }(\mathrm{kg}) / 50 \times[1-F],
\end{aligned}
$$

with $F=$ lung fraction as measured by Tc99-MAA.

The nominal target dose is the mean dose we aim to deliver to the injected liver volume, without taking in consideration the tumoral to nontumoral ratio. The common goal of glass microsphere TARE is to deliver a radiation dose of 80-150 Gy to the injected liver volume. Some authors as well as our group are using the partition model for Theraphere. This partition model provides the information of the dose delivered to the tumor. In a preliminary study, Garin et al. demonstrated that a dose above 205 Gy delivered to the tumor allowed achieving a good efficacy with a median overall survival reaching 24.5 months (95\% CI 12-28.7 months) for hepatocellular carcinoma patients with segmental or lobar portal vein thrombosis [7, 33]. This is the first publication establishing a threshold of activity delivered to the tumor as a prognostic factor, and this is only feasible using the partition model.

The main difference between glass and resin microspheres is the amount of activity per sphere, which correlates with a lower number of glass microspheres per $\mathrm{GBq}$ $\left(1.2 \times 10^{6} / 3 \mathrm{GBq}\right)$, compared with that of resin microspheres $\left(6 \times 10^{7} / 3 \mathrm{GBq}\right)$. As a result, the biologic effect and then the toxicity per Gy are lower for glass microspheres [34]. This is probably due to more homogenous distribution of resin microspheres and physical 
embolization effect. In our experience, the higher number of resin microspheres can limit the implantation of the whole prescribed activity of ${ }^{90} \mathrm{Y}$ (back flow, abdominal pain) in case of small tumors, particularly for uveal melanoma and neuroendocrine liver metastasis.

\section{How to Avoid Radioembolization Liver Insufficiency?}

The concept of radioembolization-induced liver disease (REILD) has initially been reported in 2008 by Sangro et al. [35] after having observed in 9 of 45 (20\%) consecutive patients (without chronic liver disease) a liver toxicity comparable to that previously observed after external beam radiation (called : RILD $=$ anicteric ascites) and after whole-body irradiation combined with ablative dose of chemotherapy (call combined modality-induced liver disease: CMILD = ascites and jaundice). Those patients developed a clinical picture characterized by jaundice and ascites appearing 1-3 months after TARE in the absence of tumor progression or bile duct dilatation. Liver function tests showed an increase in total bilirubin ( $>$ $3 \mathrm{mg} / \mathrm{dL}$ or $>50 \mu \mathrm{mol} / \mathrm{L}$ ) in all cases, a rising alkaline phosphatase and gamma-glutamyl transpeptidase levels and no changes in transaminases levels.

The pathological features of REILD are as for RILD, a complete obliteration of central veins, sublobular veins, and centrilobular sinusoids lumina by erythrocytes trapped in a network of reticulin and collagen, as well as congestion in peri-portal areas. These lesions lead to the hypoxic death of centrilobular hepatocytes. These features defined the venoocclusive disease (VOD). The formation of clots in the central veins and in the hepatic sinusoids could be due to a hypercoagulable state secondary to radiation-related injury to the sinusoidal endothelial cells. After approximately 4 months, the congestion resolves and the liver heals gradually; rarely fatal issues related to REILD have been reported [36]. In metastatic patients, this liver injury might also be due to previous lines of chemotherapy which sometimes lead to a sinusoidal obstructive syndrome (SOS) such as with oxaliplatin. The hallmark of SOS is the predominance of sinusoidal injuries (dilatation and engorgement) with subsequent necrosis of peri-venular hepatocytes [36]. In the landmark paper by Sangro et al. [35], the incidence of REILD was $20 \%$ in patients without chronic liver disease. The update from the same team published in 2013 [37] combining the data of 260 patients showed a decrease of REILD from 22.7 to $5.4 \%$, secondary to changes in the treatment design and in the process of activity calculation. In 2009, Kennedy et al. [31], reported the outcomes of 515 patients (680 TARE) treated in 16 centers across the United States and Europe. REILD was diagnosed in $4 \%$ of the patients. Same numbers were reported by Lam et al. [38] in a series of 247 patients. In this last report, the incidence raised to $25 \%$ for patients treated with repeated TARE to the same target volume.

Numerous risk factors associated with REILD have been described. The more frequently reported ones are listed in Table 3. Fewer data are available concerning REILD after TARE with glass microspheres but the incidence $(4 \%)$ and risk factors appear to be comparable [31, 37]. Most of the published papers reporting REILD were based on data obtained with resin microspheres. The higher number of resin microspheres needed to be injected for the same activity regarding glass microspheres could impact the hepatic arterial blood flow and consecutively the incidence of the REILD. The main risk factors are linked to the ability of the remnant or nontumoral liver to tolerate the treatment, and to the activity injected. Indeed, parameters associated with liver function such as the degree of cholestasis, the liver volume (after surgery or the percentage of tumor replacement before surgery), and the type of previous treatment (intravenous or intra-arterial chemotherapy) appear to be strong predictive factors of the risk of REILD.

The first way to avoid REILD is to limit the prescription of TARE to multidisciplinary teams in tertiary centers working with experienced interventional radiologists and nuclear medicine specialists. The second way to avoid this life-threatening complication is to strictly respect the contra-indications of TARE especially, an elevated total bilirubin value at baseline (higher than 1.5 the normal value or $>2 \mathrm{mg} / \mathrm{dL}$ ). The third way is to take into account the known risk factors by:

- avoiding whole-liver treatment and sparing as much liver as possible by sequential treatment with superselective catheterization [37] and an interval of 6 weeks between two procedures, especially for noncirrhotic patients [39].

- waiting for a 2-month chemotherapy-free interval after TARE in case of salvage approach [37].

- adapting the administered activity to the patient situation including non treated liver volume after TARE, as well as liver status (post-chemotherapy, cirrhosis): not more than $150 \mathrm{~Gy}$ if small remnant liver, important tumor involvement $(>70 \%)$ or heavily pretreated liver [31].

- avoiding repeated TARE of the same targeted liver volume [40].

- preferring TARE to chemoembolization or intra-arterial chemotherapy as initial therapy [39].

The role of the methods of radiation activity calculation (empiric, BSA or partition model) as a risk factor of REILD is still debated. Sangro et al. [37] showed that using the BSA method for whole-liver treatment and the partition model for selective treatment may lead to a decreased incidence of REILD. Salem et al. assumed that the 
BSA method instead of the empiric one enhances patient safety [31].

Several pharmacologic approaches have been assessed in order to prevent the REILD. Sangro et al. [37] suggested that taking ursodesoxycholic acid (300 $\mathrm{mg}$ bid for 2 months) in association with methyl-prednisolone $(8 \mathrm{mg}$ per day 1 month and $4 \mathrm{mg}$ once a day another month) starting the day of TARE could decrease the incidence and the severity of REILD. This approach as well as the prescription of low dose heparin or pentoxyfylline or defibrotide needs to be validated prospectively. It appears essential to mention that not all jaundice with ascites after TARE are REILD. Indeed, especially in the setting of cirrhotic patients, jaundice, ascites as well as high value of total bilirubin, alkaline phosphatase, and gamma-glutamyl transpeptidase can also be due to the evolution of the underlying liver disease even after TARE.

There is no defined treatment of REILD in the literature. Supportive medications like diurectics and symptomatic care are often proposed, Corticoids can also be used but there is no data supporting any efficacy of this option.

\section{Conclusion}

TARE is a complex procedure that necessitates the combined expertise of interventional radiologists, nuclear medicine specialists, and oncologists. The pre-treatment strategy is of crucial importance both to avoid complications and to deliver a maximal dose to the tumor sparing liver parenchyma.

Conflict of interest Alban Denys, Marc Pracht, Rafael Duran, Boris Guiu, Salim Adib, Ariane Boubaker and Pierre Bize have no conflict of interest related to this study.

\section{References}

1. Flynn WJ (1967) The treatment of pulmonary metastases with microspheres of yttrium 90. Minerva Med 58(99):4498-4500

2. Gray BN, Anderson JE, Burton MA et al (1992) Regression of liver metastases following treatment with yttrium-90 microspheres. Aust N Z J Surg 62(2):105-110

3. Shepherd FA, Rotstein LE, Houle S, Yip TC, Paul K, Sniderman KW (1992) A phase I dose escalation trial of yttrium-90 microspheres in the treatment of primary hepatocellular carcinoma. Cancer 70(9):2250-2254

4. Lau WY, Leung WT, Ho S et al (1994) Treatment of inoperable hepatocellular carcinoma with intrahepatic arterial yttrium-90 microspheres: a phase I and II study. Br J Cancer 70(5):994-999

5. Roche A, Girish BV, de Baere T et al (2003) Trans-catheter arterial chemoembolization as first-line treatment for hepatic metastases from endocrine tumors. Eur Radiol 13(1):136-140

6. Wang LM, Jani AR, Hill EJ, Sharma RA (2013) Anatomical basis and histopathological changes resulting from selective internal radiotherapy for liver metastases. J Clin Pathol 66(3):205-211
7. Garin E, Lenoir L, Rolland Y et al (2012) Dosimetry based on 99mTc-macroaggregated albumin SPECT/CT accurately predicts tumor response and survival in hepatocellular carcinoma patients treated with 90Y-loaded glass microspheres: preliminary results. J Nucl Med 53(2):255-263

8. Elschot M, Vermolen BJ, Lam MG, de Keizer B, van den Bosch MA, de Jong HW (2013) Quantitative comparison of PET and Bremsstrahlung SPECT for imaging the in vivo yttrium-90 microsphere distribution after liver radioembolization. PLoS ONE $8(2): \mathrm{e} 55742$

9. van den Hoven AF, Prince JF, Samim M et al (2014) Posttreatment PET-CT-Confirmed Intrahepatic Radioembolization Performed Without Coil Embolization, by Using the Antireflux Surefire Infusion System. Cardiovasc Intervent Radiol 37(2):523-528

10. Rose SC, Kikolski SG, Chomas JE (2013) Downstream hepatic arterial blood pressure changes caused by deployment of the surefire antireflux expandable tip. Cardiovasc Intervent Radiol 36(5):1262-1269

11. Favelier S, Germain T, Genson PY et al (2014) Anatomy of liver arteries for interventional radiology. Diagn Interv Imaging. doi:10.1016/j.diii.2013.12.001

12. Lauenstein TC, Heusner TA, Hamami M et al (2011) Radioembolization of hepatic tumors: flow redistribution after the occlusion of intrahepatic arteries. RoFo 183(11):1058-1064

13. Bilbao JI, Garrastachu P, Herraiz MJ et al (2010) Safety and efficacy assessment of flow redistribution by occlusion of intrahepatic vessels prior to radioembolization in the treatment of liver tumors. Cardiovasc Intervent Radiol 33(3):523-531

14. Burgmans MC, Kao YH, Irani FG et al (2012) Radioembolization with infusion of yttrium-90 microspheres into a right inferior phrenic artery with hepatic tumor supply is feasible and safe. J Vasc Interv Radiol 23(10):1294-1301

15. Abdelmaksoud MH, Louie JD, Kothary N et al (2011) Embolization of parasitized extrahepatic arteries to reestablish intrahepatic arterial supply to tumors before yttrium-90 radioembolization. J Vasc Interv Radiol 22(10):1355-1362

16. Hamoui N, Minocha J, Memon K et al (2013) Prophylactic embolization of the gastroduodenal and right gastric arteries is not routinely necessary before radioembolization with glass microspheres. J Vasc Interv Radiol 24(11):1743-1745

17. Hashimoto M, Heianna J, Tate E, Kurosawa R, Nishii T, Mayama I (2001) The feasibility of retrograde catheterization of the right gastric artery via the left gastric artery. J Vasc Interv Radiol 12(9):1103-1106

18. Aloia TA, Barakat O, Connelly J et al (2009) Gastric radiation enteritis after intra-arterial yttrium-90 microsphere therapy for early stage hepatocellular carcinoma. Exp Clin Transplant 7(3):141-144

19. Petroziello MF, McCann JW, Gonsalves CF et al (2011) Sidebranch embolization before $90 \mathrm{Y}$ radioembolization: rate of recanalization and new collateral development. Am J Roentgenol 197(1):W169-174

20. Samuelson SD, Louie JD, Sze DY (2013) N-butyl cyanoacrylate glue embolization of arterial networks to facilitate hepatic arterial skeletonization before radioembolization. Cardiovasc Intervent Radiol 36(3):690-698

21. Lam MG, Banerjee S, Louie JD et al (2013) Root cause analysis of gastroduodenal ulceration after yttrium-90 radioembolization. Cardiovasc Intervent Radiol 36(6):1536-1547

22. McWilliams JP, Kee ST, Loh CT, Lee EW, Liu DM (2011) Prophylactic embolization of the cystic artery before radioembolization: feasibility, safety, and outcomes. Cardiovasc Intervent Radiol 34(4):786-792

23. Hickey R, Lewandowski RJ (2011) Hepatic radioembolization complicated by radiation cholecystitis. Semin Intervent Radiol 28(2):230-233 
24. Ahmadzadehfar H, Mohlenbruch M, Sabet A et al (2011) Is prophylactic embolization of the hepatic falciform artery needed before radioembolization in patients with $99 \mathrm{mTc}-\mathrm{MAA}$ accumulation in the anterior abdominal wall? Eur J Nucl Med Mol Imaging 38(8):1477-1484

25. Sharma AK, Foster TH, Katz A, Lee DE, Waldman DL (2014) Supraumbilical rash caused by nontarget radioembolization to the falciform artery. J Vasc Interv Radiol 25(3):483-484

26. Leong QM, Lai HK, Lo RG, Teo TK, Goh A, Chow PK (2009) Radiation dermatitis following radioembolization for hepatocellular carcinoma: a case for prophylactic embolization of a patent falciform artery. J Vasc Interv Radiol 20(6):833-836

27. Wang DS, Louie JD, Kothary N, Shah RP, Sze DY (2013) Prophylactic topically applied ice to prevent cutaneous complications of nontarget chemoembolization and radioembolization. J Vasc Interv Radiol 24(4):596-600

28. Lau WY, Kennedy AS, Kim YH et al (2012) Patient selection and activity planning guide for selective internal radiotherapy with yttrium-90 resin microspheres. Int J Radiat Oncol Biol Phys 82(1):401-407

29. Giammarile F, Bodei L, Chiesa C et al (2011) EANM procedure guideline for the treatment of liver cancer and liver metastases with intra-arterial radioactive compounds. Eur J Nucl Med Mol Imaging 38(7):1393-1406

30. Kennedy A, Nag S, Salem R et al (2007) Recommendations for radioembolization of hepatic malignancies using yttrium-90 microsphere brachytherapy: a consensus panel report from the radioembolization brachytherapy oncology consortium. Int J Radiat Oncol Biol Phys 68(1):13-23

31. Kennedy AS, McNeillie P, Dezarn WA et al (2009) Treatment parameters and outcome in 680 treatments of internal radiation with resin 90Y-microspheres for unresectable hepatic tumors. Int J Radiat Oncol Biol Phys 74(5):1494-1500

32. Kao YH, Tan EH, Ng CE, Goh SW (2011) Clinical implications of the body surface area method versus partition model dosimetry for yttrium-90 radioembolization using resin microspheres: a technical review. Ann Nucl Med 25(7):455-461
33. Garin E, Lenoir L, Edeline J et al (2013) Boosted selective internal radiation therapy with 90Y-loaded glass microspheres (B-SIRT) for hepatocellular carcinoma patients: a new personalized promising concept. Eur J Nucl Med Mol Imaging 40(7):1057-1068

34. Walrand S, Hesse M, Chiesa C, Lhommel R, Jamar F (2014) The low hepatic toxicity per Gray of $90 \mathrm{Y}$ glass microspheres is linked to their transport in the arterial tree favoring a nonuniform trapping as observed in posttherapy PET imaging. J Nucl Med 55(1):135-140

35. Sangro B, Gil-Alzugaray B, Rodriguez J et al (2008) Liver disease induced by radioembolization of liver tumors: description and possible risk factors. Cancer 112(7):1538-1546

36. Guha C, Kavanagh BD (2011) Hepatic radiation toxicity: avoidance and amelioration. Semin Radiat Oncol 21(4):256-263

37. Gil-Alzugaray B, Chopitea A, Inarrairaegui M et al (2013) Prognostic factors and prevention of radioembolization-induced liver disease. Hepatology 57(3):1078-1087

38. Lam MG, Louie JD, Iagaru AH, Goris ML, Sze DY (2013) Safety of repeated yttrium-90 radioembolization. Cardiovasc Intervent Radiol 36(5):1320-1328

39. Piana PM, Gonsalves CF, Sato T et al (2011) Toxicities after radioembolization with yttrium-90 SIR-spheres: incidence and contributing risk factors at a single center. J Vasc Interv Radiol 22(10): 1373-1379

40. Lam MG, Abdelmaksoud MH, Chang DT et al (2013) Safety of $90 \mathrm{Y}$ radioembolization in patients who have undergone previous external beam radiation therapy. Int J Radiat Oncol Biol Phys 87(2):323-329

41. Medical LCS (undated) SIR-Spheres training program: physicians and institutions. Sirtex Medical

42. Dezarn WA, Cessna JT, DeWerd LA et al (2011) Recommendations of the American Association of Physicists in Medicine on dosimetry, imaging, and quality assurance procedures for $90 \mathrm{Y}$ microsphere brachytherapy in the treatment of hepatic malignancies. Med Phys 38(8):4824-4845 